Published in final edited form as:

J Am Coll Surg. 2015 December ; 221(6): 1041-1049. doi:10.1016/j.jamcollsurg.2015.09.005.

\title{
Recurrence Rate and Pattern of Perihilar Cholangiocarcinoma after Curative Intent Resection
}

\author{
Bas Groot Koerkamp, MD, PhD, \\ Department of Surgery, Memorial Sloan Kettering Cancer Center, New York, NY \\ Department of Surgery, Erasmus MC, University Medical Center Rotterdam, Rotterdam, The \\ Netherlands
}

\author{
Jimme K Wiggers, MD, \\ Department of Surgery, Academic Medical Center Amsterdam, Amsterdam, The Netherlands \\ Peter J Allen, MD, FACS, \\ Department of Surgery, Memorial Sloan Kettering Cancer Center, New York, NY
}

Marc G Besselink, MD, PhD,

Department of Surgery, Academic Medical Center Amsterdam, Amsterdam, The Netherlands

Leslie H Blumgart, MD, FACS,

Department of Surgery, Memorial Sloan Kettering Cancer Center, New York, NY

Olivier RC Busch, MD, PhD,

Department of Surgery, Academic Medical Center Amsterdam, Amsterdam, The Netherlands

Robert J Coelen, MD,

Department of Surgery, Academic Medical Center Amsterdam, Amsterdam, The Netherlands

Michael I D'Angelica, MD, FACS,

Department of Surgery, Memorial Sloan Kettering Cancer Center, New York, NY

Ronald P DeMatteo, MD, FACS,

Department of Surgery, Memorial Sloan Kettering Cancer Center, New York, NY

Dirk J Gouma, MD, PhD,

Department of Surgery, Academic Medical Center Amsterdam, Amsterdam, The Netherlands

T Peter Kingham, MD, FACS,

Department of Surgery, Memorial Sloan Kettering Cancer Center, New York, NY

William R Jarnagin, MD, FACS, and

Correspondence address: Bas Groot Koerkamp, MD, PhD. Department of Surgery, Erasmus MC, P.O. Box 2040, 3000 CA,

Rotterdam, The Netherlands. Telephone: +31-6-33330948, b.grootkoerkamp@erasmusmc.nl.

Drs Koerkamp and Wiggers contributed equally to this work.

Publisher's Disclaimer: This is a PDF file of an unedited manuscript that has been accepted for publication. As a service to our customers we are providing this early version of the manuscript. The manuscript will undergo copyediting, typesetting, and review of the resulting proof before it is published in its final form. Please note that during the production process errors may be discovered which could affect the content, and all legal disclaimers that apply to the journal pertain.

Disclosure Information: Nothing to disclose.

Presented at the 11th Congress of the European-African Hepato-Pancreato-Biliary Association, Manchester, UK, April 2015. 
Department of Surgery, Memorial Sloan Kettering Cancer Center, New York, NY

Thomas M van Gulik, MD, PhD

Department of Surgery, Academic Medical Center Amsterdam, Amsterdam, The Netherlands

\section{Abstract}

Background-To investigate the rate and pattern of recurrence after curative intent resection of perihilar cholangiocarcinoma ( $\mathrm{PHC})$.

Study design-Patients were included from two prospectively maintained databases.

Recurrences were categorized by site. Time to recurrence and recurrence free survival (RFS) were estimated using the Kaplan-Meier method. Cox proportional hazards modeling was used to identify independent poor prognostic factors.

Results-Between 1991 and 2012, 306 consecutive patients met the inclusion criteria. The median overall survival was 40 months. A recurrence was diagnosed in 177 patients (58\%). An initial local recurrence was found in $26 \%$ of patients: liver hilum (11\%), hepaticojejunostomy $(8 \%)$, liver resection margin (8\%), or distal bile duct remnant (2\%). An initial distant recurrence was observed in $40 \%$ of patients: retroperitoneal lymph nodes (14\%), intrahepatic away from the resection margin (13\%), peritoneum (12\%), and lungs (8\%). Only $18 \%$ of patients had an isolated initial local recurrence. The estimated overall recurrence rate was $76 \%$ at 8 years. After a recurrence free period of 5 years, $28 \%$ of patients developed a recurrence in the next 3 years. Median RFS was 26 months. Independent prognostic factors for RFS were resection margin, lymph node status, and tumor differentiation. Only node-positive PHC precluded RFS beyond 7 years.

Conclusions-PHC will recur in most patients (76\%) after resection, emphasizing the need for better adjuvant strategies. The high recurrence rate up to eight years justifies prolonged surveillance. Only patients with an isolated initial local recurrence (18\%) may have benefited from a more extensive resection or liver transplantation. Node-positive PHC appears incurable.

\section{INTRODUCTION}

Perihilar cholangiocarcinoma (PHC) is the most common type of bile duct cancer with an annual incidence of 1 to 2 per 100,000 in Western countries. PHC arises at or near the confluence of the right and left main bile duct and has an American Joint Committee on Cancer (AJCC) staging separate from intrahepatic and distal cholangiocarcinoma.(1) Patients typically present with obstructive jaundice and are staged with cross-sectional imaging of the abdomen and chest. In the absence of metastatic or locally advanced disease, patients are eligible for resection with curative intent.(2) Five-year overall survival (OS) after resection varies from $13 \%$ to $40 \%$ across series with more than 100 patients.(3)

The poor 5-year OS reflects a high recurrence rate after curative intent resection of PHC. Although several studies have reported the probability of developing a recurrence(4-8), only one study with 79 patients evaluated the time to recurrence.(9) Detailed patterns of recurrence were reported in only one study with 80 patients.(8) Better knowledge about recurrence rate and pattern may improve preoperative, intraoperative, and postoperative 
decision making. For example, surgery may not be the optimal treatment for patients with a very poor recurrence free survival (RFS), patients with a high risk of isolated local (without distant) recurrence may benefit from more extensive surgery or liver transplantation, and patients with a high risk of distant recurrence may be more likely to benefit from adjuvant treatment. Moreover, postoperative surveillance could be tailored to recurrence rate and pattern.

The objective of this study was to investigate the rate and pattern of recurrence after curative intent resection of PHC. In addition, prognostic factors for the time to recurrence and RFS were evaluated.

\section{METHODS}

\section{Patients}

From 1991 to 2012, consecutive patients with resected PHC were included from two prospectively maintained databases from Memorial Sloan Kettering Cancer Center (MSKCC, New York, USA), and Academic Medical Center (AMC, Amsterdam, The Netherlands). Patients with postoperative mortality within 90 days, R2 (palliative) resection, and re-resection after prior resection at another hospital were excluded from the analyses. Patients who were found to have a diagnosis other than PHC at final pathologic review were also excluded. The institutional review board (IRB) at both institutions approved this study.

Patients underwent resection if tumor-free proximal and distal bile ducts were anticipated with no metastases beyond the hepatoduodenal ligament. Suspect lymph nodes beyond the hepatoduodenal ligament (ie N2 lymph nodes) were biopsied with endoscopic ultrasound fine needle aspiration or percutaneous biopsy preoperatively, or analyzed with frozen section intraoperatively. Resections typically included excision of the extrahepatic bile duct en bloc with an (extended) hemihepatectomy, excision of the portal vein bifurcation when involved, and complete lymphadenectomy of the hepatoduodenal ligament. Routine frozen section was performed of proximal and distal bile duct margins. In selected patients an extrahepatic bile duct resection without liver resection was performed. Caudate lobectomy was performed in most patients since the late 90s, and in most patients with a left-sided partial hepatectomy. Patients treated at AMC underwent preoperative low-dose radiotherapy with three fractions of $3.5 \mathrm{~Gy}$ in an effort to destruct tumor cells in bile and reduce postoperative seeding metastases.(10) Perioperative patient management and patient selection for surgery have been described previously in more detail. $(11,12)$

\section{Outcomes}

All patients underwent surveillance for recurrence. Imaging (CT or MRI) was performed at the discretion of the physician and patient because there is no data to support aggressive surveillance. In accordance with the National Comprehensive Cancer Network (NCCN) guidelines, imaging was performed at prespecified intervals (typically 6 months) or only with clinical suspicion of recurrence.(13) The use of imaging at regular intervals was more common in MSKCC and in more recent years. 
Recurrence was defined as any new lesion on imaging that was highly suspicious for recurrence of PHC. Pathologic confirmation was often obtained, but not required. In a previous analysis in 2003, recurrence patterns were categorized into local, regional, and distant.(8) In the current study, the term "regional recurrence" has been abandoned. Regional recurrence referred to recurrence in retroperitoneal lymph nodes, including celiac, periaortic, and pericaval nodes. Since the $7^{\text {th }}$ edition of the AJCC staging system was published these nodes are classified as N2 representing stage IV disease.(1) Consequently, recurrences in retroperitoneal lymph nodes are now classified as distant metastases. In addition, a new local recurrence category was introduced for patients with a recurrence in the distal common bile duct remnant. Local recurrence was now defined as any recurrence at the liver resection margin, distal bile duct remnant, hepaticojejunostomy, or elsewhere in the liver hilum (e.g., hilar lymph nodes or arising from perineural disease). All other recurrences were considered distant.

Time to initial recurrence was measured from the time of surgery to the time of first recurrence whether local, distant, or both simultaneously. Patients who had no observed recurrence were censored at the time of last follow-up. Patients who died from other causes were censored at the time of death. Patients who died from sequelae of PHC without a documented recurrence were censored at the time of last follow-up prior to death. These patients either died from an undocumented recurrence, or from late complications resulting from biliary drainage or surgery. Recurrence free survival (RFS) was measured from the time of surgery to the time of first recurrence or death from any cause. Survival status was determined from national registries. Patients who were alive without evidence of recurrence were censored at the time of last follow-up.

\section{Variables}

Poor prognostic factors for time to recurrence and RFS were selected based on literature review. $(6,14-17)$ Patients with a negative margin were divided between a narrow and a wide margin. A narrow margin was defined as an initial positive margin (typically on frozen section), but a negative definitive margin with additional resection.(18) Lymph node status was divided into three groups: positive, negative with less than 4 lymph nodes evaluated, and negative with at least 4 nodes evaluated.(19, 20) Moderate and poor tumor differentiation were combined because OS and RFS were similar for these groups.

\section{Statistical analyses}

Statistical analyses were performed using SPSS (Statistical Package for the Social Sciences), version 22. Proportions were compared with Fischer exact or chi square test; means were compared with the t-test. Time to recurrence and RFS were estimated using the KaplanMeier method. Univariable analyses were conducted using Kaplan-Meier estimates of survival probabilities and the log-rank test for comparisons. Variables with a p-value less than 0.05 were entered into a cox proportional hazards regression modeling for multivariable analyses for both time to recurrence and RFS. 


\section{RESULTS}

\section{Patients}

Between 1991 and 2012, 359 consecutive patients underwent a resection for PHC of whom 306 patients (173 from MSKCC, 133 from AMC) met the inclusion criteria. Table 1 presents patient characteristics and treatments including demographics, preoperative imaging, biliary drainage, surgical procedure, pathology, and adjuvant treatments. Median overall survival (OS) was 40 months (95\% CI: 35-46 months) and 5-year OS was 35\%. The 10 -year OS was $17 \%$ with 24 actual 10-year survivors. Patients with a node negative tumor and a wide negative resection margin $(\mathrm{n}=129,42 \%)$ had a median OS of 63 months and 5year OS of 50\%. During follow-up, 214 patients (70\%) died. PHC was the cause of death for 195 patients (91\%): 177 had a documented recurrence, 18 patients died from an undocumented recurrence or from late complications resulting from biliary drainage or surgery. Nineteen patients (9\%) died of other causes. The median follow-up for patients alive at last follow-up was 48 months.

\section{Time to initial recurrence}

During follow-up 177 patients (58\%) were diagnosed with a recurrence. Figure 1A presents the estimated cumulative probability of recurrence over time. The median time to recurrence was 31 months. At two years follow-up, the estimated probability of a recurrence was $42 \%$, at five years $67 \%$, and at eight years $76 \%$. Of the patients who did not develop a recurrence during the first two years, $60 \%$ developed a recurrence before eight years of follow-up. Of the patients who did not develop a recurrence during the first five years, $28 \%$ developed a recurrence before eight years of follow-up. Only two patients developed a recurrence after more than eight years.

\section{Site of recurrence}

Table 2 presents the site of initial recurrence. An initial local recurrence was found in 81 (26\%) and an initial distant recurrence in 123 (40\%) of all 306 patients. Only 54 patients (18\% of all patients) had an initial isolated local recurrence, that is, without a simultaneously diagnosed distant recurrence.

The probability of an initial isolated local recurrence was $14 \%$ in patients with a wide negative resection margin, and $21 \%$ in patients with a narrow or positive margin $(\mathrm{p}=0.22)$. Caudate resection was not associated with initial isolated local recurrence $(\mathrm{p}=0.64)$ or a negative resection margin $(\mathrm{p}=0.39)$. Liver recurrence was less common at the resection margin representing local recurrence ( $8 \%$ of all patients) than intrahepatically representing distant recurrence (13\% of all patients). Of the eight patients with an initial abdominal wall recurrence, five patients (1.6\%) had an initial isolated abdominal wall recurrence, all at the site of the laparotomy scar. An initial isolated abdominal wall recurrence was not associated with biliary drainage approach: two patients underwent preoperative percutaneous biliary drainage, and three patients endoscopic drainage only.

Time to recurrence was similar for patients with an initial local or distant recurrence $(\mathrm{p}=0.46)$. Most patients with a time to recurrence of more than 5 years $(\mathrm{n}=15)$ had an initial 
distant recurrence without a preferred distant site. Median OS after recurrence was 8 months, and was also similar for patients with local and distant recurrence $(\mathrm{p}=0.91)$. OS at two years after diagnosis of a recurrence was $18 \%$ and at three years $5 \%$.

\section{Prognostic factors for time to recurrence}

Figures 1B-D present the univariable probability of recurrence over time for the key prognostic factors. The independent poor prognostic factors for the time to recurrence were positive lymph nodes (HR 2.62, 95\% CI, 1.90-3.63), evaluation of less than 4 lymph nodes (HR 1.39, 95\% CI, 1.03-1.88), a positive or narrow resection margin (HR 1.51, 95\% CI 1.12-2.04), and a poor or moderate tumor differentiation (HR 2.25, 95\% CI 1.47-3.42). Independent prognostic factors for the time to distant recurrence were the same factors as for any recurrence. Independent prognostic factors for the time to an initial isolated local recurrence were positive lymph node status (HR 2.65, 95\%, 1.48-4.69) and a positive or narrow resection margin (HR 1.95, 95\% CI, 1.14-3.34).

\section{Recurrence free survival}

The median recurrence free survival (RFS) for all patients was 26 months (95\% CI, 21-31). The 5-year RFS was 27\% and the 10-year RFS 14\%. Univariable analyses (Table 1) identified several poor prognostic factors for a poor RFS: male gender, preoperative bilirubin above $2 \mathrm{mg} / \mathrm{dL}$, preoperative biliary drainage, positive or narrow resection margin, positive lymph node(s), less than 4 lymph nodes evaluated, moderate or poor tumor differentiation, non-papillary tumor, perineural invasion, lymphovascular invasion, T-stage 3 or 4, and AJCC stage III or IV. Adjuvant chemotherapy and radiotherapy were both associated with a very poor RFS as explained by their strong associations with node-positive and margin-positive disease. Multivariable analysis identified four independent prognostic factors for a poor RFS: a positive or narrow resection margin, positive lymph node(s), less than four lymph nodes evaluated, and moderate or poor tumor differentiation (Table 3). All other variables were not associated with RFS in multivariable analysis; caudate resection was not a prognostic factor for RFS. RFS beyond 7 years was not observed in 78 patients with node-positive disease, with only 6 patients censored due to incomplete follow-up. All other poor prognostic factors did not preclude RFS beyond 7 years.

\section{DISCUSSION}

At eight years after resection of PHC in 306 patients, we found an estimated recurrence rate of $76 \%$. Five other studies with each more than 100 patients observed recurrence probabilities of $49 \%$ to $66 \%$ (Table 4). However, observed recurrence probabilities typically underestimate the actual recurrence rate due to incomplete follow-up. Only one other study used survival analysis to account for incomplete follow-up.(9) This study was limited to patients with margin-negative resection and recurrence rate estimates up to four years only. At four years the recurrence rate for patients with a negative-margin was similar to the present study (Figure 1B). However, we demonstrated that patients continue to have recurrences beyond four years follow-up until a plateau is reached at about eight years. 
The high rate of recurrence up to eight years may justify prolonged postoperative surveillance. The NCCN guideline currently recommends considering surveillance imaging every six months for two years.(13) We demonstrated that about half of the initial recurrences are diagnosed after this two year period. While this could justify surveillance beyond two years, it remains unclear whether early detection and treatment of a recurrence improves outcome. The efficacy of palliative chemotherapy has been demonstrated for patients with unresectable or metastatic cholangiocarcinoma, but this efficacy may not transfer to the setting of recurrence after a curative intent resection. $(21,22)$ Moreover, it remains to be determined whether patients with an initial isolated local recurrence benefit from early detection and re-resection or radiotherapy. Surveillance with CT of the chest could potentially benefit only $8 \%$ of patients in whom we found an initial recurrence in the lungs or mediastinum.

An initial isolated local recurrence was found in only $18 \%$ of patients. Three studies with more than 100 patients reported an initial isolated local recurrence in $4 \%$ to $11 \%$ (Table 4). Kobayashi et al. found an initial isolated local recurrence in $10 \%$ of 78 margin-negative patients.(9) In the present study, we classified retroperitoneal nodal recurrences as distant recurrences in accordance with the $7^{\text {th }}$ edition of the AJCC staging system.(1) The study of Jarnagin et al. was performed prior to this $7^{\text {th }}$ edition and classified retroperitoneal nodal recurrences as locoregional recurrences, resulting in a higher estimate for initial isolated locoregional recurrence.(8) The current definition of local recurrence appears appropriate because it includes the area that is typically resected for PHC. Local recurrences arise because microscopic disease is left behind inadvertently at the liver resection margin, distal bile duct remnant, hepaticojejunostomy, or anywhere else in the liver hilum. These truly local recurrences could in theory be avoided by more extensive resections. Neuhaus et al. have proposed a "hilar en bloc resection" involving a combined extended right hepatectomy with main portal vein resection in all patients.(23) Unfortunately, this procedure has a high postoperative mortality and can only potentially benefit those $18 \%$ of patients with an isolated initial local recurrence. A hepatopancreatoduodenectomy has been advocated to avoid local recurrence at the distal bile duct remnant but is also associated with an increased postoperative mortality.(24) The increased postoperative mortality after more extensive resections should be weighed against the potential reduction in local recurrence rate. Total hepatectomy with liver transplantation is the most extensive resection to avoid local recurrence. In a multicenter series of 287 transplanted PHC patients, only $20 \%$ of patients had a recurrence after transplant (sites unspecified) with an RFS of $59 \%$ at 10 years, compared to $14 \%$ in the present study.(25) However, the transplant study involved highly selected patients of whom $63 \%$ had primary sclerosing cholangitis (PSC) as underlying disease, only $6 \%$ had positive lymph nodes, and $10 \%$ underwent an additional pancreatoduodenectomy. Following the strict criteria for liver transplantation for PHC (node-negative, tumor diameter $<3 \mathrm{~cm}$, and stage T4 or underlying PSC), only 13 patients $(4 \%)$ of the present study cohort would have qualified. Nevertheless, transplant outcomes compare favorably to resection even after excluding patients with PSC or node-positive disease.

Kobayashi et al. found a recurrence rate at 3 years of $80 \%$ in node-positive patients.(9) This was similar to the present study, but Figure 1C demonstrates that the recurrence rate 
continues to rise and approximates $100 \%$ with adequate follow-up. RFS beyond 7 years was not observed in 78 patients with node-positive disease. It appears from this study that node positive PHC cannot be cured with resection alone. Unfortunately, preoperative imaging has a low accuracy in detecting lymph node metastasis, so selection of patients with nodepositive disease for alternative treatment strategies is not feasible with current imaging modalities.(26) Patients with node-positive PHC may still benefit from a resection by resolving biliary obstruction and avoiding recurrent cholangitis associated with biliary drainage. Moreover, while cure may be unlikely, a resection in node-positive patients could still improve life expectancy. For example, although these results should be interpreted carefully due to selection bias, a recent study from Nagoya showed that survival in 120 patients who underwent resection of N1 node-positve PHC was worse compared to survival in 174 patients who underwent resection of node-negative PHC, but still better compared to survival of 118 patients who underwent surgery without resection.(19) However, these potential benefits should be weighed against a substantial risk of postoperative mortality of $10-11 \%$ in two large Western nation-wide cohorts and from $0 \%$ to $14 \%$ in a review of highvolume centers. $(3,6,27,28)$ Extensive resections with increased mortality to obtain clear margins are probably not justified in all patients with node-positive PHC.

Univariable analyses of RFS identified many poor prognostic factors. Node-positive disease was the most important independent poor prognostic factor, for both distant and local recurrence. More than half of the node-negative patients had less than 4 lymph nodes evaluated. Consequently, many of these node-negative patients may have harbored undetected positive lymph nodes, as reflected by the worse RFS as compared to nodenegative patients with at least 4 lymph nodes evaluated. Evaluating at least 4 lymph nodes is unlikely to cure node-positive patients, but has been recommended for adequate staging.(29) Tumor diameter on imaging or pathologic examination was not associated with RFS, contrary to findings in the liver transplant literature were transplant for PHC is not recommended for tumors of more than $3 \mathrm{~cm}$ in diameter.(25) This may be explained by differences in patient and tumor characteristics as mentioned above.

This study has several limitations. Firstly, surveillance imaging was at the discretion of the physician and patient, in accordance with the NCCN guidelines and justified by the absence of data to support aggressive surveillance. Imaging was performed at regular intervals in some patients and only when symptoms developed in other patients.(13) This has likely resulted in an underestimate of the time to recurrence and the RFS. However, because of adequate follow-up this probably had little impact on outcomes at 5 or 8 years. Moreover, the interval between recurrence showing up on imaging and causing symptoms can only be a few months, based on the median OS of 8 months after developing a recurrence. Secondly, an inherent limitation of time to recurrence analysis is that patients who die from other causes are censored at the time of death. The underlying assumption is that patients who died from other causes are lost from follow-up at random at the time of death. These patients may have been more or less likely to develop a recurrence if they would not have died. However, in the present study the resulting bias is probably small, because only 19 patients (7\%) died of other causes. 
In summary, most patients recur after a curative intent resection of PHC, emphasizing the need for better adjuvant strategies. The high rate of recurrences up to 8 years may justify prolonged postoperative surveillance. Only patients with an isolated initial local recurrence (18\%) could have benefited from a more extensive resection or liver transplantation, and future studies should aim to better identify these patients preoperatively. The presence of positive lymph nodes was the only poor prognostic factor precluding RFS beyond seven years. Node-positive PHC appears incurable with currently available treatment modalities.

\section{Acknowledgments}

Support: Dr Groot Koerkamp was funded by the Dutch Cancer Society, grant number UVA 2011-4973. Dr Wiggers was funded by the Academic Medical Center Foundation. This study was supported in part by NIH/NCI P30 CA008748 (Cancer Center Support Grant).

\section{REFERENCES}

1. Edge, SB. AJCC Cancer Staging Manual. 7th. New York, USA: Springer; 2010.

2. Jarnagin WR, Fong Y, DeMatteo RP, et al. Staging, resectability, and outcome in 225 patients with hilar cholangiocarcinoma. Ann Surg. 2001; 234:507-517. discussion 517-519. [PubMed: 11573044]

3. Popescu I, Dumitrascu T. Curative-intent surgery for hilar cholangiocarcinoma: prognostic factors for clinical decision making. Langenbeck's Arch Surg / Deutsche Gesellschaft fur Chirurgie. 2014; 399:693-705.

4. Chen XP, Lau WY, Huang ZY, et al. Extent of liver resection for hilar cholangiocarcinoma. Br J Surg. 2009; 96:1167-1175. [PubMed: 19705374]

5. Dumitrascu T, Chirita D, Ionescu M, Popescu I. Resection for hilar cholangiocarcinoma: analysis of prognostic factors and the impact of systemic inflammation on long-term outcome. J Gastrointest Surg. 2013; 17:913-924. [PubMed: 23319395]

6. Nuzzo G, Giuliante F, Ardito F, et al. Improvement in perioperative and long-term outcome after surgical treatment of hilar cholangiocarcinoma: results of an Italian multicenter analysis of 440 patients. Arch Surg. 2012; 147:26-34. [PubMed: 22250108]

7. Wahab MA, Sultan AM, Salah T, et al. Caudate lobe resection with major hepatectomy for central cholangiocarcinoma: is it of value? Hepato-Gastroenterol. 2012; 59:321-324.

8. Jarnagin WR, Ruo L, Little SA, et al. Patterns of initial disease recurrence after resection of gallbladder carcinoma and hilar cholangiocarcinoma: implications for adjuvant therapeutic strategies. Cancer. 2003; 98:1689-1700. [PubMed: 14534886]

9. Kobayashi A, Miwa S, Nakata T, Miyagawa S. Disease recurrence patterns after R0 resection of hilar cholangiocarcinoma. Br J Surg. 2010; 97:56-64. [PubMed: 19937985]

10. Gerhards MF, Gonzalez DG, ten Hoopen-Neumann H, et al. Prevention of implantation metastases after resection of proximal bile duct tumours with pre-operative low dose radiation therapy. Eur J Surg Oncol. 2000; 26:480-485. [PubMed: 11016470]

11. Matsuo K, Rocha FG, Ito K, et al. The Blumgart preoperative staging system for hilar cholangiocarcinoma: analysis of resectability and outcomes in 380 patients. J Am Coll Surg. 2012; 215:343-355. [PubMed: 22749003]

12. van Gulik TM, Kloek JJ, Ruys AT, et al. Multidisciplinary management of hilar cholangiocarcinoma (Klatskin tumor): extended resection is associated with improved survival. Eur J Surg Oncol. 2011; 37:65-71. [PubMed: 21115233]

13. National Comprehensive Cancer Network. Hepatobiliary Cancers. 2015 Available at: http:// www.nccn.org/professionals/physician_gls/pdf/hepatobiliary.pdf.

14. Furusawa N, Kobayashi A, Yokoyama T, et al. Surgical treatment of 144 cases of hilar cholangiocarcinoma without liver-related mortality. World J Surg. 2014; 38:1164-1176. [PubMed: 24305942] 
15. Klempnauer J, Ridder GJ, von Wasielewski R, et al. Resectional surgery of hilar cholangiocarcinoma: a multivariate analysis of prognostic factors. J Clin Oncol. 1997; 15:947954. [PubMed: 9060532]

16. Lee SG, Song GW, Hwang S, et al. Surgical treatment of hilar cholangiocarcinoma in the new era: the Asan experience. J Hepato-Biliary-Pancreatic Sci. 2010; 17:476-489.

17. Song SC, Choi DW, Kow AW, et al. Surgical outcomes of 230 resected hilar cholangiocarcinoma in a single centre. ANZ J Surg. 2013; 83:268-274. [PubMed: 22943422]

18. Endo I, House MG, Klimstra DS, et al. Clinical significance of intraoperative bile duct margin assessment for hilar cholangiocarcinoma. Ann Surg Oncol. 2008; 15:2104-2112. [PubMed: 18543039]

19. Aoba T, Ebata T, Yokoyama Y, et al. Assessment of nodal status for perihilar cholangiocarcinoma: location, number, or ratio of involved nodes. Ann Surg. 2013; 257:718-725. [PubMed: 23407295]

20. Guglielmi A, Ruzzenente A, Campagnaro T, et al. Patterns and prognostic significance of lymph node dissection for surgical treatment of perihilar and intrahepatic cholangiocarcinoma. $\mathrm{J}$ Gastrointest Surg. 2013; 17:1917-1928. [PubMed: 24048613]

21. Valle J, Wasan H, Palmer DH, et al. Cisplatin plus gemcitabine versus gemcitabine for biliary tract cancer. N Engl J Med. 2010; 362:1273-1281. [PubMed: 20375404]

22. Valle JW, Furuse J, Jitlal M, et al. Cisplatin and gemcitabine for advanced biliary tract cancer: a meta-analysis of two randomised trials. Ann Oncol. 2014; 25:391-398. [PubMed: 24351397]

23. Neuhaus $\mathrm{P}$, Thelen A, Jonas S, et al. Oncological superiority of hilar en bloc resection for the treatment of hilar cholangiocarcinoma. Ann Surg Oncol. 2012; 19:1602-1608. [PubMed: 21964888]

24. Kaneoka Y, Yamaguchi A, Isogai M, Kumada T. Survival benefit of hepatopancreatoduodenectomy for cholangiocarcinoma in comparison to hepatectomy or pancreatoduodenectomy. WorldJ Surg. 2010; 34:2662-3670. [PubMed: 20607255]

25. Darwish Murad S, Kim WR, Harnois DM, et al. Efficacy of neoadjuvant chemoradiation, followed by liver transplantation, for perihilar cholangiocarcinoma at 12 US centers. Gastroenterology. 2012; 143:88-98. e3; quiz e14. [PubMed: 22504095]

26. Ruys AT, van Beem BE, Engelbrecht MR, et al. Radiological staging in patients with hilar cholangiocarcinoma: a systematic review and meta-analysis. Br J Radiol. 2012; 85:1255-1262. [PubMed: 22919007]

27. Kaiser GM, Paul A, Sgourakis G, et al. Novel prognostic scoring system after surgery for Klatskin tumor. Am Surg. 2013; 79:90-95. [PubMed: 23317618]

28. Farges O, Regimbeau JM, Fuks D, et al. Multicentre European study of preoperative biliary drainage for hilar cholangiocarcinoma. Br J Surg. 2013; 100:274-283. [PubMed: 23124720]

29. Ito K, Ito H, Allen PJ, et al. Adequate lymph node assessment for extrahepatic bile duct adenocarcinoma. Ann Surg. 2010; 251:675-681. [PubMed: 20224368] 


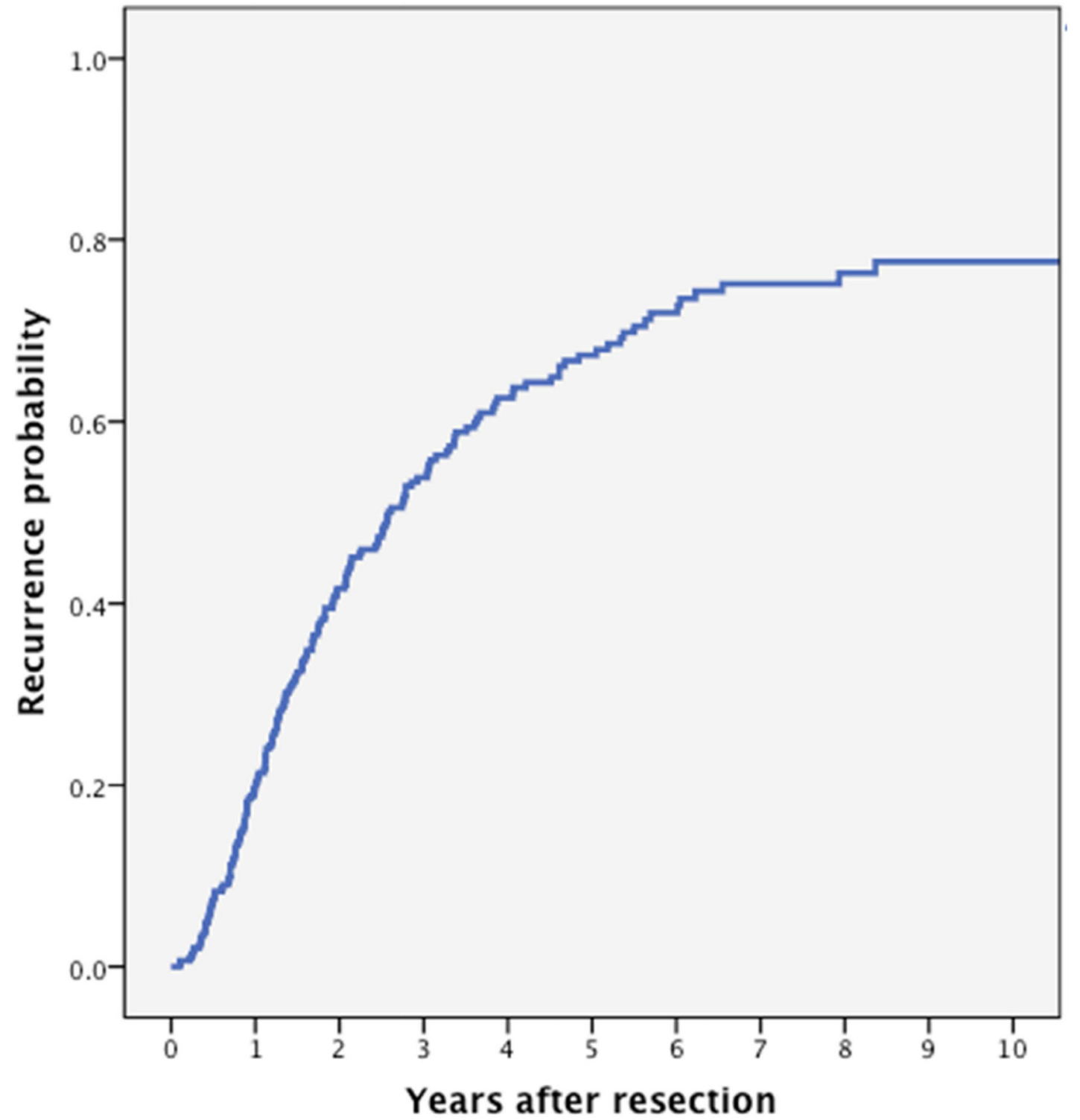

\begin{tabular}{|l|l|l|l|l|l|l|l|l|l|l|l|}
\hline Number at risk & 306 & 239 & 150 & 107 & 77 & 60 & 43 & 29 & 20 & 19 & 14 \\
\hline
\end{tabular}



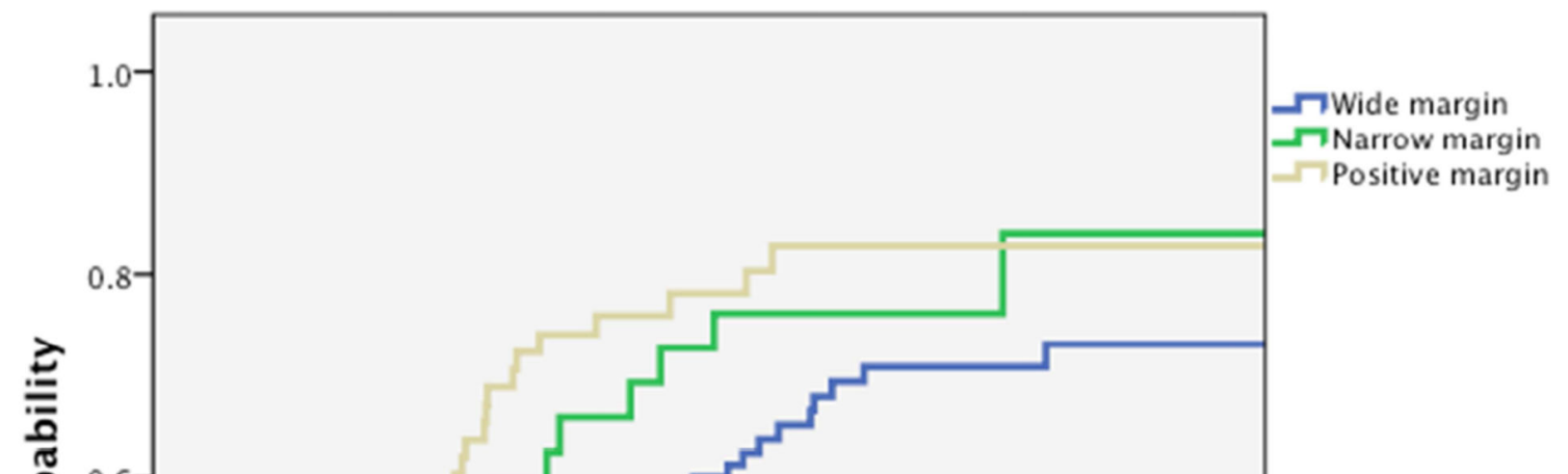

\begin{tabular}{|l|l|l|l|l|l|l|l|l|l|l|l|}
\hline Wide margin & 168 & 137 & 92 & 73 & 51 & 39 & 29 & 18 & 13 & 12 & 8 \\
\hline Narrow margin & 54 & 45 & 27 & 14 & 12 & 10 & 8 & 5 & 3 & 3 & 2 \\
\hline Positive margin & 84 & 55 & 30 & 19 & 14 & 10 & 6 & 6 & 4 & 4 & 4 \\
\hline
\end{tabular}

B 

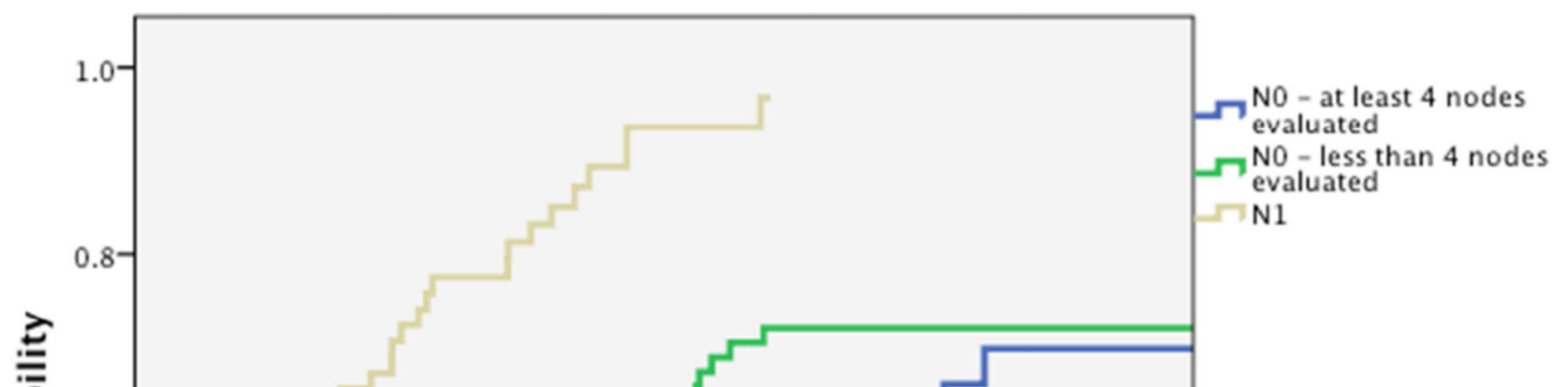

Years after resection

\begin{tabular}{|l|l|l|l|l|l|l|l|l|l|l|l|}
\hline N0, $>=4$ nodes & 104 & 89 & 59 & 45 & 30 & 26 & 17 & 12 & 9 & 7 & 5 \\
\hline N0, $<4$ nodes & 124 & 104 & 69 & 49 & 40 & 31 & 24 & 17 & 11 & 11 & 9 \\
\hline N1 & 78 & 44 & 20 & 12 & 7 & 3 & 2 & 0 & 0 & 0 & 0 \\
\hline
\end{tabular}

C 


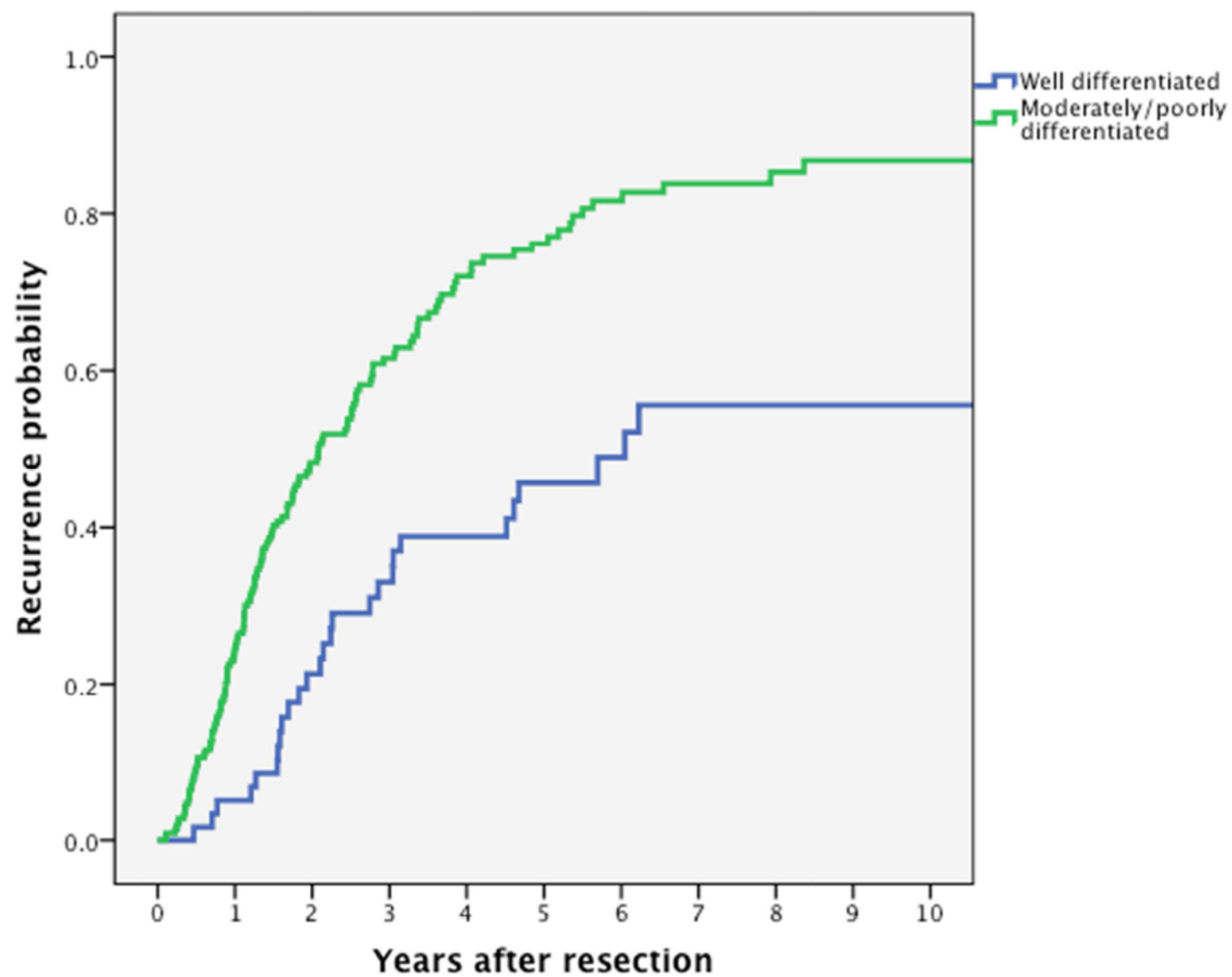

\begin{tabular}{|l|l|l|l|l|l|l|l|l|l|l|l|}
\hline Well & 68 & 60 & 46 & 37 & 35 & 27 & 22 & 13 & 10 & 10 & 8 \\
\hline Moderate/poor & 228 & 170 & 96 & 62 & 37 & 29 & 18 & 14 & 10 & 9 & 6 \\
\hline
\end{tabular}

Figure 1.

Cumulative probability of recurrence (local or distant) after curative intent resection for perihilar cholangiocarcinoma with number of patients at risk. (A) All patients; (B) margin wide, narrow, or positive, $\mathrm{p}=0.002$; (C) N0 (node-negative with at least 4 evaluated lymph nodes), $\mathrm{Nx}$ (node-negative with less than 4 evaluated lymph nodes), vs N1 (node-positive), $\mathrm{p}<0.001$; (D) well versus moderately or poorly differentiated tumor, $\mathrm{p}<0.001$. 
Table 1

Patient Characteristics and Univariable Analyses for Recurrence-Free Survival

\begin{tabular}{|c|c|c|c|c|}
\hline & n $(\%)$ & $\begin{array}{l}\text { Median } \\
\text { RFS, mo }\end{array}$ & $\underset{(\%)}{5-y \text { RFS }}$ & $\begin{array}{c}\text { p Value, } \\
\text { univariable }\end{array}$ \\
\hline All patients & 306 & 26 & 27 & - \\
\hline Sex & & & & 0.04 \\
\hline Female & $127(42)$ & 33 & 30 & \\
\hline Male & $179(58)$ & 24 & 21 & \\
\hline Age y & & & & 0.77 \\
\hline$<65$ & $160(52)$ & 25 & 25 & \\
\hline$\checkmark 65$ & $146(48)$ & 29 & 30 & \\
\hline PSC & & & & -- \\
\hline Yes & $4(1)$ & -- & -- & \\
\hline No & $302(99)$ & -- & -- & \\
\hline Bilirubin, preoperative, $\mathrm{mg} / \mathrm{dL}$ & & & & 0.006 \\
\hline$<2$ & $186(65)$ & 35 & 31 & \\
\hline$\geq 2$ & $99(35)$ & 20 & 18 & \\
\hline Lobar atrophy on imaging & & & & 0.10 \\
\hline None & $188(66)$ & 27 & 26 & \\
\hline Left & $59(20)$ & 24 & 27 & \\
\hline Right & $39(14)$ & 33 & 35 & \\
\hline Bismuth classification on imaging & & & & 0.10 \\
\hline Left or right duct only & $23(8)$ & 62 & 56 & \\
\hline 1 & $57(19)$ & 21 & 21 & \\
\hline 2 & $40(13)$ & 33 & 27 & \\
\hline $3 \mathrm{~A}$, right & $79(26)$ & 25 & 23 & \\
\hline $3 \mathrm{~B}$, left & $64(21)$ & 25 & 29 & \\
\hline 4 & $43(14)$ & 24 & 28 & \\
\hline Portal vein involvement on imaging & & & & 0.68 \\
\hline None & $176(63)$ & 26 & 27 & \\
\hline Main/bifurcation/bilateral & $8(3)$ & 49 & 30 & \\
\hline Left & $57(20)$ & 24 & 30 & \\
\hline Right & $38(14)$ & 27 & 17 & \\
\hline Suspected lymph node involvement on imaging ${ }^{* \dagger}$ & & & & 0.30 \\
\hline Yes & $166(63)$ & 31 & 30 & \\
\hline No & $97(37)$ & 21 & 24 & \\
\hline Blumgart classification on imaging & & & & 0.57 \\
\hline $\mathrm{T} 1$ & $144(47)$ & 25 & 26 & \\
\hline $\mathrm{T} 2$ & $94(31)$ & 29 & 29 & \\
\hline $\mathrm{T} 3$ & $47(15)$ & 25 & 28 & \\
\hline
\end{tabular}




\begin{tabular}{|c|c|c|c|c|}
\hline & n $(\%)$ & $\begin{array}{l}\text { Median } \\
\text { RFS, mo }\end{array}$ & $\underset{(\%)}{\text { 5-y RFS }}$ & $\begin{array}{c}\text { p Value, } \\
\text { univariable }\end{array}$ \\
\hline Tumor diameter on imaging, $\mathrm{cm}$ & & & & 0.96 \\
\hline$<3$ & $179(69)$ & 23 & 24 & \\
\hline 23 & $80(31)$ & 33 & 24 & \\
\hline Drainage, preoperative & & & & 0.02 \\
\hline None & $60(20)$ & 47 & 36 & \\
\hline Percutaneous & $35(11)$ & 23 & 19 & \\
\hline Endoscopic & $157(51)$ & 29 & 30 & \\
\hline Both & $54(18)$ & 19 & 17 & \\
\hline Type of resection & & & & 0.44 \\
\hline Bile duct resection alone & $53(17)$ & 23 & 18 & \\
\hline Right hemihepatectomy & $25(8)$ & 23 & 34 & \\
\hline Left hemihepatectomy & $86(28)$ & 25 & 33 & \\
\hline Extended right hepatectomy & $89(29)$ & 26 & 24 & \\
\hline Extended left hepatectomy & $27(9)$ & 44 & 42 & \\
\hline Segment $4 \mathrm{~b} / 5$ resection & $24(8)$ & 30 & 23 & \\
\hline Central hepatectomy & $2(1)$ & -- & -- & \\
\hline Caudate resection & & & & 0.67 \\
\hline Yes & $137(54)$ & 26 & 30 & \\
\hline No & $116(46)$ & 29 & 26 & \\
\hline Resection margin & & & & 0.002 \\
\hline Positive & $84(27)$ & 19 & 15 & \\
\hline Narrow & $54(18)$ & 24 & 26 & \\
\hline Wide & $168(55)$ & 39 & 34 & \\
\hline Lymph node involvement & & & & $<0.001$ \\
\hline Yes & $78(26)$ & 13 & 5 & \\
\hline No, less than 4 nodes & $124(41)$ & 31 & 30 & \\
\hline No, at least 4 nodes & $104(34)$ & 42 & 41 & \\
\hline Tumor differentiation & & & & $<0.001$ \\
\hline Well & $68(23)$ & 56 & 48 & \\
\hline Moderate or poor & $228(77)$ & 22 & 19 & \\
\hline Perineural invasion & & & & 0.004 \\
\hline Yes & $211(69)$ & 22 & 23 & \\
\hline No & $95(31)$ & 40 & 37 & \\
\hline Lymphovascular invasion & & & & 0.008 \\
\hline Yes & $79(26)$ & 20 & 18 & \\
\hline No & $227(74)$ & 31 & 30 & \\
\hline Papillary tumor & & & & 0.005 \\
\hline Yes & $57(19)$ & 55 & 47 & \\
\hline No & $249(81)$ & 23 & 23 & \\
\hline
\end{tabular}




\begin{tabular}{|l|c|c|c|c|}
\hline & $\mathbf{n}(\boldsymbol{\%})$ & $\begin{array}{c}\text { Median } \\
\text { RFS, mo }\end{array}$ & $\begin{array}{c}\text { 5-y RFS } \\
(\%)\end{array}$ & $\begin{array}{c}\text { p Value, } \\
\text { univariable }\end{array}$ \\
\hline T-stage $-7^{\text {th }}$ edition & & & & 0.02 \\
\hline T0-2 & $232(76)$ & 31 & 30 & \\
\hline T3-4 & $74(24)$ & 20 & 17 & \\
\hline AJCC stage $-7^{\text {th }}$ edition & & & & $<0.001$ \\
\hline 0 & $11(4)$ & 55 & 50 & \\
\hline 1 & $35(11)$ & 58 & 48 & \\
\hline 2 & $133(43)$ & 33 & 32 & \\
\hline 3 & $78(26)$ & 16 & 12 & \\
\hline 4 & $49(16)$ & 19 & 18 & \\
\hline Adjuvant chemotherapy & & & & $<0.001$ \\
\hline Yes & $24(8)$ & 13 & 0 & \\
\hline No & $282(92)$ & 29 & 29 & \\
\hline Adjuvant radiotherapy & & & & $<0.001$ \\
\hline Yes & $25(8)$ & 15 & 0 & \\
\hline No & $282(92)$ & 29 & 30 & \\
\hline
\end{tabular}

Tumor diameter and suspected lymph node involvement on imaging were missing in $47(15 \%)$ and 42 patients (14\%) respectively, because they were missing in the report and imaging from the early nineties was not always available for review.

${ }^{\dagger}$ Suspected lymph node involvement on imaging was defined as short-axis diameter larger than $10 \mathrm{~mm}$ or central necrosis.

* The results presented for caudate resection concerns only patients who had a liver resection. PSC, primary sclerosing cholangitis; RFS, recurrence free survival. 
Table 2

Initial Recurrence after Resection of Perihilar Cholangiocarcinoma

\begin{tabular}{|c|c|c|c|}
\hline Site & $\begin{array}{l}\text { No. of } \\
\text { recurrences }\end{array}$ & $\begin{array}{l}\text { All } 177 \text { patients with } \\
\text { a recurrence, \%* }\end{array}$ & $\begin{array}{c}\text { All 306 } \\
\text { patients, \% }\end{array}$ \\
\hline \multicolumn{4}{|l|}{ Local } \\
\hline Liver hilum & 34 & 19 & 11 \\
\hline Hepaticojejunostomy & 23 & 13 & 8 \\
\hline Distal bile duct remnant & 6 & 3 & 2 \\
\hline Liver resection margin & 23 & 13 & 8 \\
\hline \multicolumn{4}{|l|}{ Distant } \\
\hline Liver intrahepatic & 40 & 23 & 13 \\
\hline Retroperitoneal lymph nodes ${ }^{\dagger}$ & 42 & 24 & 14 \\
\hline Peritoneum* & 38 & 21 & 12 \\
\hline Lung or mediastinum & 25 & 14 & 8 \\
\hline Abdominal wall/incision & 8 & 5 & 2 \\
\hline Bone & 3 & 2 & 1 \\
\hline Skin & 1 & 1 & 0 \\
\hline Adrenal gland & 1 & 1 & 0 \\
\hline Axillary or neck lymph nodes & 2 & 1 & 1 \\
\hline Spleen & 1 & 1 & 0 \\
\hline \multicolumn{4}{|l|}{ Totals } \\
\hline Recurrences, no. & 245 & & \\
\hline Patients with a recurrence, $\mathrm{n}$ & 177 & & 58 \\
\hline Patients with an intial local recurrence, $n$ & 81 & & 26 \\
\hline Patients with an initial isolated local recurrence, $\mathrm{n}$ & 54 & & 18 \\
\hline Patients with an initial distal recurrence, $\mathrm{n}$ & 123 & & 40 \\
\hline
\end{tabular}

Note that patients can have an initial recurrence at more than one site. Consequently, total percentage of recurrence across all sites exceeds $100 \%$.

* Includes positive cytology of ascites and omental metastases.

${ }^{\dagger}$ Includes periaortic, pericaval, and celiac artery lymph nodes. 
Table 3

Multivariable Analysis for Recurrence-Free Survival

\begin{tabular}{|l|l|l|l|}
\hline Prognostic factor & HR & 95\% CI for HR & $\begin{array}{l}\text { Multivariable p } \\
\text { value }\end{array}$ \\
\hline Margin positive & 1.42 & $1.05-1.91$ & 0.02 \\
\hline Lymph node positive & 3.06 & $2.15-4.34$ & $<0.001$ \\
\hline$<4$ lymph nodes evaluated & 1.46 & $1.05-2.04$ & 0.03 \\
\hline Moderate or poor differentiation & 1.97 & $1.40-2.79$ & $<0.001$ \\
\hline
\end{tabular}




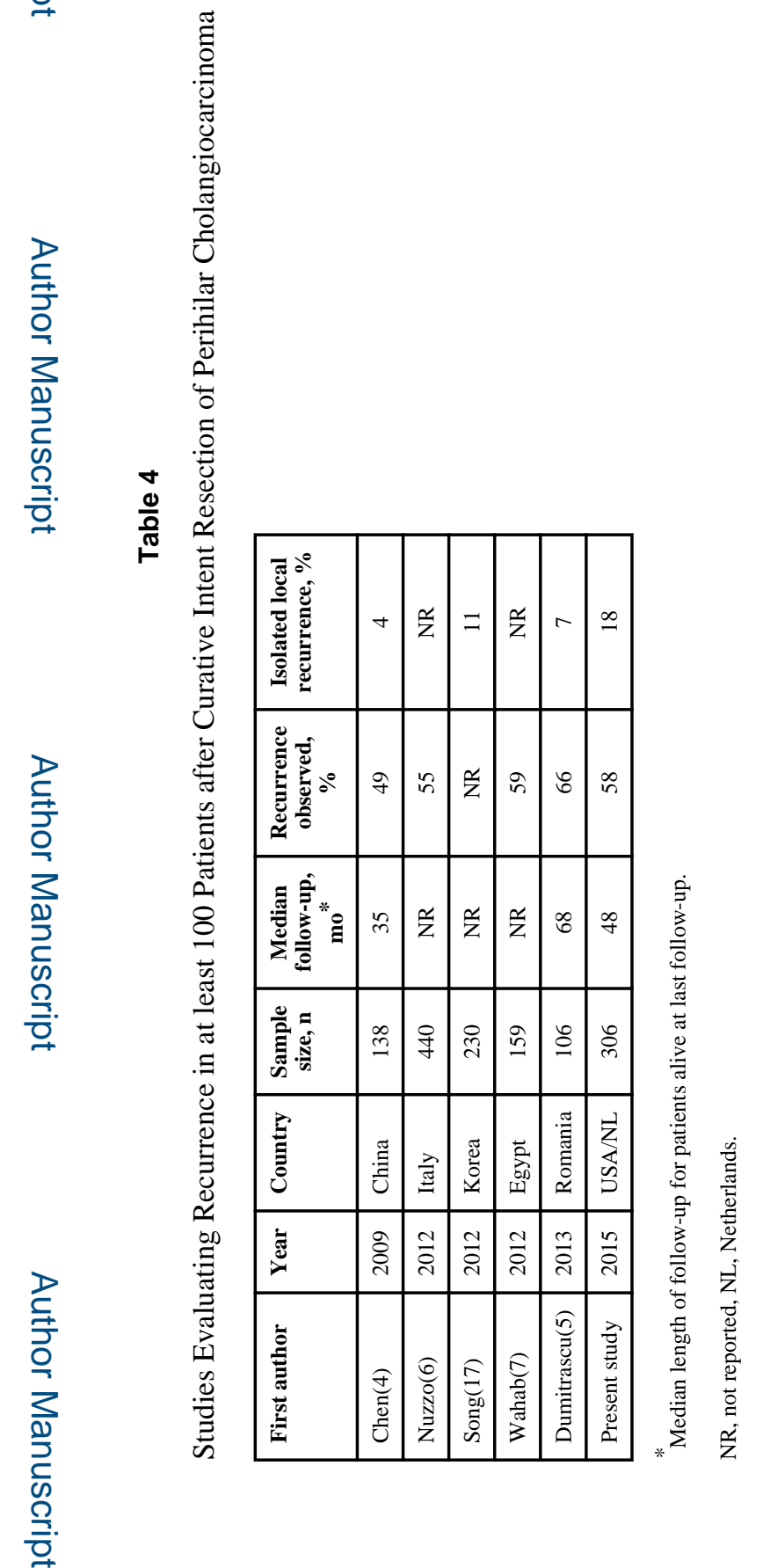

$J$ Am Coll Surg. Author manuscript; available in PMC 2016 December 01. 